\title{
El conocimiento especializado del docente de matemáticas
}

\author{
Francisco Javier Hernández Gutiérrez \\ Eugenio Lizarde Flores
}

Escuela Normal Rural "Gral. Matías Ramos Santos"

\section{Resumen}

1 objetivo de este estudio es ca$\checkmark$ racterizar el Conocimiento Especializado del Profesor de Matemáticas (Carrillo, y otros, 2014) en el proceso de formación inicial de los futuros docentes de educación primaria en la Escuela Normal Rural "Gral. Matías Ramos Santos" en Zacatecas, México. Para ello, se realizó un estudio estadístico correlacional de Pearson con la aplicación de un cuestionario con reactivos Likert. La muestra consistió en 52 alumnos que cursan el cuarto semestre de la licenciatura. Los resultados mostraron una relación estadísticamente significativa en las correlaciones tanto en los niveles de dificultad de la enseñanza de los contenidos de matemáticas como en su aprendizaje.

Palabras clave: Conocimiento especializado, formación de docentes

\section{Introducción}

Este estudio forma parte de otro más amplio en el que se pretende caracterizar el Conocimiento Especializado del Profesor de Matemáticas (MTSK por sus siglas en inglés) (Carrillo, Climent, Contreras, \& $\mathrm{Mu}$ ñoz-Catalán, 2013)(Carrillo, y otros, 2014), principalmente en la vertiente de la formación inicial, es decir, cómo se plantea que se construya desde las propuestas curriculares oficiales (DOF, 2012) y (SEP, 2012), con la posibilidad de valorar los alcances y limitaciones de éstas, así como las eventuales transformaciones que se generan tanto en las concepciones iniciales de los futuros docentes, como en el dominio disciplinario y la configuración del conocimiento especializado, bajo la consideración de que lo "especializado" alude a lo que es necesario y específico a la enseñanza de la matemática. 
En el presente estudio se reportan los resultados de la aplicación de una encuesta a una muestra compuesta por 52 alumnos que cursan el $4^{\circ}$ semestre de la LEP, tomando como foco de análisis los subdominios KOT, KFLM y KMLS, con lo cual se cubren a su vez el dominio matemático y el didáctico comprendidos en el MTSK.

Aunque ésta contempló 56 variables, aquí reportamos los resultados del análisis de correlación de Pearson entre el nivel de dificultad de la enseñanza y aprendizaje que los estudiantes normalistas conciben al "didactificar" los 28 problemas matemáticos relacionados a los 8 temas del Programa de Estudio de matemáticas para sexto grado (SEP, 2012).

Los resultados indican que hay una correlación significativa entre las concepciones que el docente asume de la dificultad para que los alumnos aprendan el contenido y sus propias dificultades para desarrollar estrategias de enseñanza, es decir, en la medida que asumen un contenido difícil para aprender por parte de los niños, al mismo tiempo asumen que les representará mayores dificultades en su enseñanza; al parecer estas concepciones se generan desde la constatación de sus propias dificultades para resolver los mismo problemas matemáticos a los que se enfrentan.

\section{Planteamiento del problema}

Los resultados internacionales en competencia matemática, como los que reporta la prueba PISA 2012, muestran que México se encuentra en el lugar 53 de 65 países evaluados. El porcentaje de los niveles de desempeño en nuestro país, indican una alta concentración en el segundo y tercer nivel con $32 \%$ y $28 \%$ respectivamente, en contra de sus porcentajes más bajos en los niveles más altos de desempeño, con un $13 \%$ en el cuarto nivel, un $4 \%$ en el quinto nivel y $0 \%$ en el sexto nivel (INEE, 2013). Esta problemática se sigue presentando de manera recurrente y nos señala poner atención en la formación inicial de profesores.

El debate actual (NCTM, 2015; Carrillo, Climent, Contreras, \& Muñoz-Catalán, 2013) en torno a la formación matemática de los profesores, incluye la discusión respecto a la pertinencia de que el maestro sea un especialista en la materia, es decir, tal y como sucede en otros países donde primero se es Licenciado en Matemáticas (formación disciplinar) y luego se recibe habilitación para ejercer la docencia; sin embargo nosotros asumimos que, más que ser "especialista", el profesor debe tener un conocimiento "especializado", sobre todo cuando nos referimos a la formación matemática de los profesores de educación primaria, como es el caso de los resultados de investigación que aquí reportamos.

Ahora bien, ¿a qué nos referimos con "conocimiento especializado"? al igual que Shulman (1986) el MTsk, como marco teórico para caracterizar el conocimiento especializado del profesor de matemáticas (Carrillo, Climent, Contreras, \& MuñozCatalán, 2013; Carrillo, Contreras, \& Flores, 2013; Carrillo, y otros, 2014; Montes, y otros, 2015) considera dos grandes dominios: El conocimiento matemático como disciplina científica que se utiliza por parte del docente en un contexto escolar y, el Conocimiento didáctico del contenido como 
los aspectos relacionados con el contenido matemático como objeto de los procesos de enseñanza y aprendizaje.

Estos dos dominios a su vez cuentan con subdominios. El conocimiento matemático se subdivide en Conocimiento de los temas matemáticos (кот), se refiere al conocimiento que el docente tiene sobre los contenidos que desarrolla con sus alumnos; Conocimiento de la estructura matemática (KsM), contempla el conocimiento que le posibilita al profesor enseñar los temas matemáticos como fundamentación para su complejidad posterior y; Conocimiento de la práctica matemática (KPM), establece la relación entre el conocimiento de los temas matemáticos y los procedimientos y prácticas que se realizan para su construcción.

En el dominio Conocimiento didáctico del contenido se establecieron los subdominios: Conocimiento de las características del aprendizaje (KFLM), maneja las características de aprendizaje que conlleva el aprendizaje en los contenidos específicos de las matemáticas; Conocimiento de la enseñanza de la matemática (ктм), se refiere a los recursos, materiales, estrategias didácticas y metodológicas como se presenta el contenido, y; Conocimiento de los estándares de aprendizaje de las Matemáticas (KMLs), se enfoca a la intencionalidad y conocimiento del profesor sobre los niveles de logro en los aprendizajes de los alumnos considerando el momento escolar determinado y su grado de desarrollo.

La aportación de la teoría del MTSK a este estudio, se enfoca en la caracterización y búsqueda de predictores para encontrar sus correlaciones estadísticas de los subdominios: Conocimiento de los temas matemáticos (кот), Conocimiento de las características del aprendizaje (KFLM) y Conocimiento de los estándares de aprendizaje de las Matemáticas (KMLs), desde las concepciones de los docentes en formación del cuarto semestre de Licenciatura en educación primaria.

Es importante considerar otros dos elementos en el proceso de análisis de las concepciones de los futuros profesores y de su configuración en el conocimiento especializado para la enseñanza de las matemáticas en la educación primaria. Primero, estudios como el de (Lizarde, 2013) y (Hernández \& Lizarde, 2015) demuestran la modificación en las concepciones de los futuros docentes vinculada con la práctica del conocimiento matemático y su didactificación a lo largo de su formación en la escuela normal; y segundo, la articulación entre las concepciones "oficiales" que permean los programas de estudio de matemáticas (SEP, 2012) y su análisis en los programas de matemáticas de la Escuela Normal.

\section{Metodología}

\section{Perfil y delimitación de los participantes}

La población objeto de estudio fue de 60 casos con posibilidad de una muestra mínima de 52, estando la proporción de la muestra $\mathrm{p}$ entre \pm 0.05 de la proporción $\mathrm{P}$ de la población con un $95 \%$ de nivel de confianza de acuerdo con Krejcie y Morgan (1970). En la aplicación concreta de la encuesta se contó con la aportación de la muestra mínima (52 casos) que corresponde al $86.6 \%$ de la población total. 
Este estudio se desarrolló con la participación de alumnos de Licenciatura en Educación Primaria de la Normal Rural "Gral. Matías Ramos Santos" de San Marcos, Loreto, Zacatecas. Las muestras se centraron en dos grupos de cuarto semestre, se consideró así porque en este semestre se cierra la preparación en matemáticas y su enseñanza y aprendizaje en los cursos de la malla curricular.

\section{Diseño del instrumento}

El instrumento para la recolección de datos que se utilizó para este estudio fue una encuesta elaborada por los autores del artículo. Los argumentos teóricos considerados para su elaboración, surgieron de la propuesta de la teoría del MTSK, con la intención de recuperar lo referente a los subdominios: Conocimiento de las características del aprendizaje (кFLM) у Conocimiento de los estándares de aprendizaje de las Matemáticas (KMLs), relacionándolos con el actual Plan y Programas de Estudio para Educación Primaria 2011 y el Plan para formación de Licenciados en Educación Primaria 2012 en México.

El instrumento se elaboró a partir de 28 problemas matemáticos extraídos de los ocho temas matemáticos establecidos en el libro de texto de matemáticas para sexto grado de educación primaria: Números y sistemas de numeración, Problemas aditivos, Problemas multiplicativos, Figuras y cuerpos geométricos, Ubicación espacial, Medida, Proporcionalidad y funciones y Análisis y representación de datos. Constó de cuatro variables nominales para la caracterización de los respondientes y 56 variables ordinales, en el caso de estas últi- mas, la forma de responder fue a través de una variación de la escala Likert con cinco opciones para responder del 1 al 5, que corresponden al grado de dificultad que el normalista otorga a la enseñanza y aprendizaje del problema planteado. Los valores asumidos fueron: muy fácil (1), fácil (2), relativamente difícil (3), difícil (4) y muy difícil (5).

Dentro de las propiedades psicométricas del instrumento, se realizó una exploración de la consistencia interna del instrumento, utilizando el método de Alfa de Cronbach, del que se obtuvo un coeficiente total de confiabilidad para las 56 variables ordinales de .941 y una correlación interítem de .233, puntaje que supera el criterio de Rosenthal (1994) y Cadena (2007).

\section{Procedimiento}

Con la finalidad de encontrar la correlación entre el nivel de dificultad de la enseñanza y aprendizaje que los estudiantes normalistas conciben al didactificar los 28 problemas matemáticos relacionados con los ocho temas del Programa de Estudio de matemáticas para sexto grado, se desarrolló un análisis de correlación de Pearson de dos listas a un nivel de significancia de $\mathrm{p} \leq .050$. Se transformaron las 56 variables ordinales en 16 variables complejas, que corresponden a los ocho temas matemáticos, midiendo en cada caso, tanto la dificultad en su enseñanza, como de su aprendizaje: Tema números y sistemas de numeración enseñanza, Tema números y sistemas de numeración aprendizaje, Tema problemas aditivos enseñanza, Tema problemas aditivos aprendizaje, Tema problemas 
multiplicativos enseñanza, Tema problemas multiplicativos aprendizaje, Tema figuras $y$ cuerpos geométricos enseñanza, Tema figuras y cuerpos geométricos aprendizaje, Tema ubicación espacial enseñanza, Tema ubicación espacial aprendizaje, Tema Medida enseñanza, Tema Medida aprendizaje, Tema proporcionalidad y funciones enseñanza, Tema proporcionalidad y funciones aprendizaje, Tema Análisis y representación de datos enseñanza y Tema Análisis y representación de datos aprendizaje.

Las características que se consideraron para el uso de la prueba paramétrica correlacional fueron: $\mathrm{N}$ superior a 30 casos, nivel de medición numérica en las variables, una distribución normal en los datos de los 56 reactivos considerados a partir de su agrupación en 16 variables complejas, utilizando la prueba estadística de Kolmorov-Smirnov y; la homocedasticidad de las varianzas en las mismas a partir de un análisis estadístico de Levene, considerando un nivel de probabilidad $\geq 0.050$ en estos dos últimos casos. Con respecto a la normalidad de las varianzas, la evidencia demuestra que se acepta hipótesis nula en 13 de las 16 variables complejas. Con respecto a la homogeneidad de las varianzas, el resultado permite manifestar que las varianzas de las variables se observan como homogéneas y por lo tanto tienen homocedasticidad y se acepta la posibilidad del uso de estadística paramétrica (Tabla 1).

Tabla 1. Prueba de Levene para homogeneidad y Prueba Kolmorov-Smirnov para normalidad de varianzas.

\begin{tabular}{|l|l|l|l|l|}
\hline Variables complejas & $\begin{array}{l}\text { Prueba de } \\
\text { Levene }\end{array}$ & $\begin{array}{l}\text { Prueba } \\
\text { Kolmorov- } \\
\text { Smirnov }\end{array}$ & & \\
\hline & F & p. & ZK-S & p. \\
\hline Tema números y sistemas de numeración enseñanza & .479 & .492 & .710 & .694 \\
\hline Tema números y sistemas de numeración aprendizaje & 2.354 & .131 & .821 & .510 \\
\hline Tema problemas aditivos enseñanza & .572 & .453 & .680 & .745 \\
\hline Tema problemas aditivos aprendizaje & .850 & .361 & 1.093 & .183 \\
\hline Tema problemas multiplicativos enseñanza & 3.895 & .054 & 1.000 & .270 \\
\hline Tema problemas multiplicativos aprendizaje & 1.975 & .166 & 1.361 & .049 \\
\hline Tema figuras y cuerpos geométricos enseñanza & 1.163 & .286 & 1.812 & .003 \\
\hline Tema figuras y cuerpos geométricos aprendizaje & .145 & .705 & 1.377 & .045 \\
\hline Tema ubicación espacial enseñanza & .132 & .718 & .981 & .291 \\
\hline Tema ubicación espacial aprendizaje & 2.185 & .146 & 1.110 & .170 \\
\hline Tema Medida enseñanza & .551 & .462 & .587 & .881 \\
\hline Tema Medida aprendizaje & .075 & .785 & .581 & .888 \\
\hline Tema proporcionalidad y funciones enseñanza & 1.254 & .268 & .984 & .288 \\
\hline Tema proporcionalidad y funciones aprendizaje & .014 & .906 & .982 & .290 \\
\hline Tema Análisis y representación de datos enseñanza & .005 & .947 & .824 & .505 \\
\hline Tema Análisis y representación de datos aprendizaje & 2.032 & .160 & .723 & .673 \\
\hline
\end{tabular}


Para correr el análisis de método de Alfa de Cronbach, la Correlación Inter-Ítem y Correlación de Pearson se utilizó el paquete estadístico Statistica 7; para la prueba de homogeneidad y normalidad de las varianzas, se utilizó el software estadístico SPSS 21.

\section{Resultados}

Los resultados demuestran niveles estadísticamente significativos en las correlaciones, es decir, existe una verdadera relación distinta a cero en todas las variables complejas sobre los temas de estudio de matemáticas en las que el futuro maestro indicó el nivel de dificultad que implicaría su enseñanza, obtuvo una correlación significativa con relación a la dificultad sobre el mismo tema en su aprendizaje por parte del alumno de sexto grado de educación primaria.

La variable compleja Tema números y sistemas de numeración enseñanza tuvo una correlación de 0.72 con respecto a su homóloga en el aprendizaje, en el caso de Tema problemas aditivos enseñanza su co- rrelación fue de 0.71 con Tema problemas aditivos aprendizaje, Tema problemas multiplicativos enseñanza 0.77 con Tema problemas multiplicativos aprendizaje, Tema figuras y cuerpos geométricos enseñanza 0.84 con la variable de aprendizaje, la correlación más fuerte se observó en el Tema ubicación espacial enseñanza 0.88 con Tema ubicación espacial aprendizaje, Tema Medida enseñanza 0.76 con Tema Medida aprendizaje, Tema proporcionalidad y funciones enseñanza 0.81 con la variable de aprendizaje y Tema Análisis y representación de datos enseñanza 0.86 de correlación con su variable compleja de aprendizaje (Figuras 1-8).

En términos generales el estudio indicó correlaciones positivas en todas las variables, además se observa la correlación significativa entre la dificultad expuesta para la enseñanza de los temas matemáticos, con la dificultad expuesta por el futuro maestro de educación primaria sobre el mismo tema pero en su aprendizaje por parte de los niños de sexto grado.
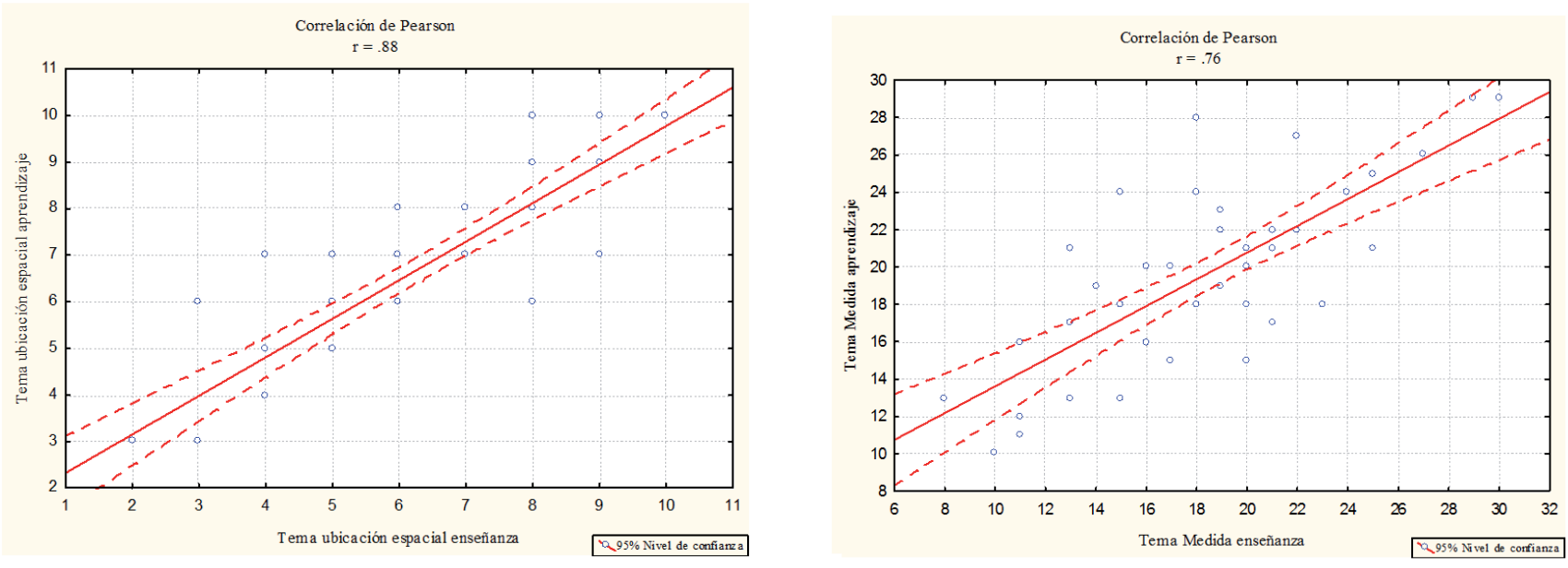
REVISTA DE INVESTIGACIÓN EDUCATIVA DE LA REDIECH N. 11
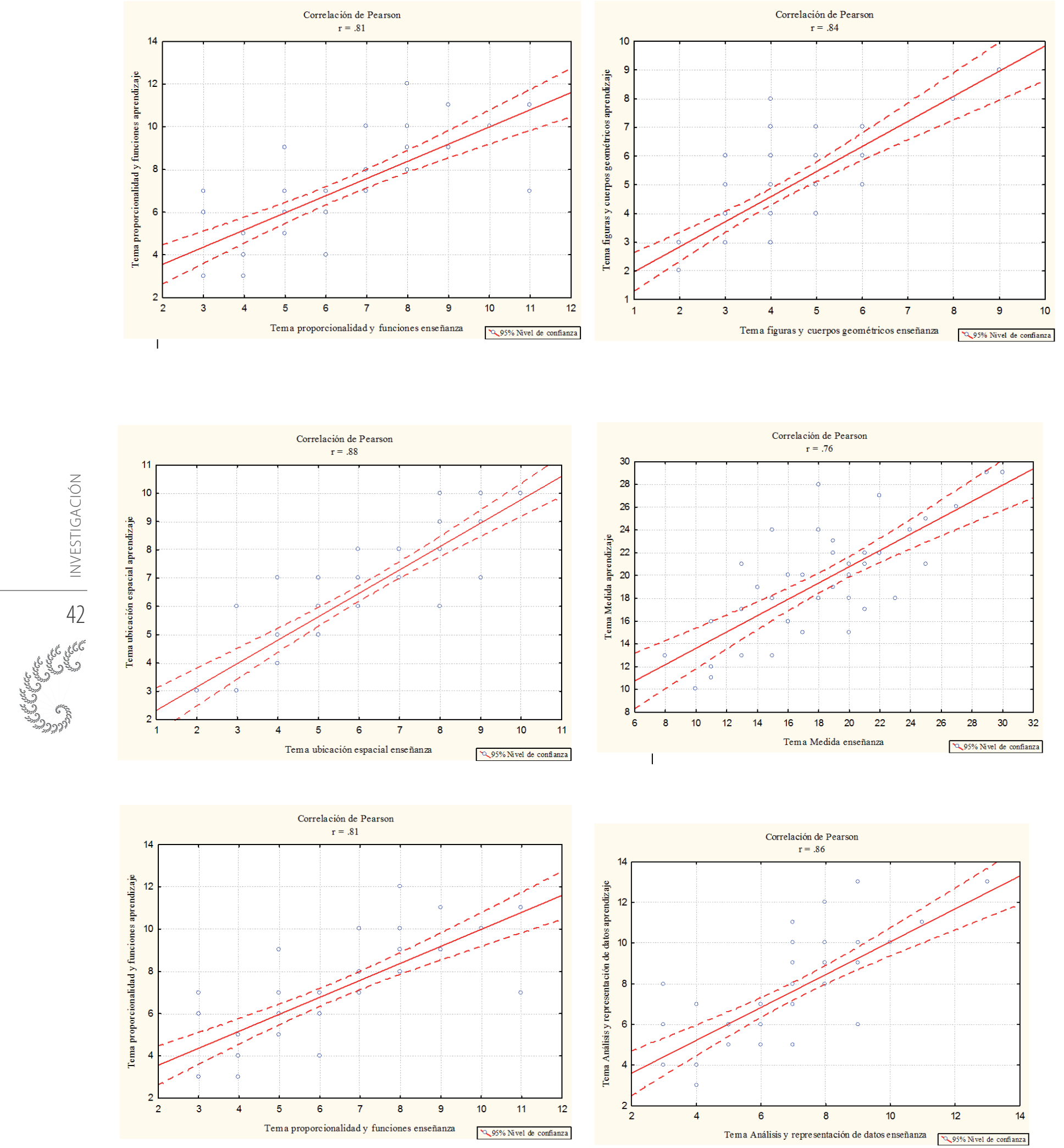

Figuras 1-8. Gráficos de Correlación de Pearson de las variables complejas 


\section{Conclusiones}

La caracterización del conocimiento especializado del profesor de matemáticas de educación primaria en formación inicial, muestra a partir del estudio desarrollado, la confirmación de relación entre dos de los subdominios del MTSK con el centro que marca la propia teoría. Es decir, las concepciones del docente manifiestan que existe un vínculo significativo entre El conocimiento de los estándares de aprendizaje de las matemáticas (KMLS) con El conocimiento de las características del aprendizaje de las matemáticas (KFLM). El análisis demuestra una correlación significativa entre las variables complejas, en este caso, las que miden los niveles de dificultad que asume el futuro maestro sobre alguno de los temas matemáticos al enseñarlo, así como la dificultad que cree que tendrá sobre ese mismo tema el aprendizaje del niño de sexto grado.

Por lo que se confirma la relación intrínseca que el docente en formación otorga tanto a la dificultad en la enseñanza de contenidos, como en su aprendizaje. De tal forma que si el estudiante normalista cree que se le dificultará didactificar un contenido específico de matemáticas, también será complicado en el mismo nivel para el alumno y, de igual forma, que si al futuro maestro se le facilita dicho contenido, al alumno de sexto grado le será más sencillo aprenderlo.

No en un sentido unilateral de que si el maestro no conoce algo, el alumno tampoco lo hará, pero sí, en el sentido de una intencionalidad didáctica, en la que entre mayor integración e intención tenga el docente de las características del conocimiento matemático y su didactificación, habrá una mejor respuesta en el proceso de enseñanza y aprendizaje de las matemáticas.

\section{Referencias Bibliográficas}

Cadena G. C.H. (2007). La medición en las ciencias sociales y psicología. En R. Landero y M. González (Comps.). Estadística con SPSS y metodología de la investigación. pp. 139-165. Trillas: México.

Carrillo, J., Climent, N., Contreras, L. C., \& MuñozCatalán, M. C. (2013). Determining Specialised Knowledge for Mathematics Teaching. En B. Ubuz, C. Haser, \& M. A. Mariotti (Ed.). Actas del CERME 8. pp. 2985-2994. Antalya: Turquía.

Carrillo, J., Contreras, L., \& Flores, P. (2013). Un modelo de conocimiento especializado del profesor de matemáticas. En L. Rico, M. Cañadas, J. Gutiérrez, M. Molina, \& I. Segovia (Ed.). Investigación en didáctica de la matemática. Homenaje a Encarnación Castro. pp. 193-200. Comares: Granada

Carrillo, J., Contreras, L. C., Climent, N., Escudero-Ávila, D., Flores-Medrano, E., \& Montes, M. Á. (2014). Un marco teórico para el conocimiento especializado del profesor de matemáticas. Universidad de Huelva publicaciones:Huelva.

DOF (2012). Acuerdo 649 por el que se establece el Plan de Estudios para la formación de maestros de educación primaria. México.

INEE (2013). México en PISA 2012. México.

Hernández, G. F., \& LizArde, E. (2015). Las concepciones de enseñanza y aprendizaje de las matemáticas de los profesores de primaria en formación inicial. Presentada en XIV CIAEM. CIAEM: Chiapas.

Krejcie, R. V. \& Morgan, D. W. (1970). Determining sample size for research activities. Educational and psychological measurement. 30. 607-610.

Lizarde, E. (2013). Transposición y destransposición del saber matemático y didáctico. Representaciones y prácticas en la formación inicial de docentes. Servicio de publicaciones de la UHU: Huelva.

Montes, M. Á., Contreras, L. C., Liñan, M. D., MuÑoz-Catalán, M. C., Climent, N., \& Carrillo, J. (2015). Conocimiento de aritmética de futuros maestros. Debilidades y fortalezas. Revista de Educación 367. pp. 36-62. 
REVISTA DE INVESTIGACIÓN EDUCATIVA DE LA REDIECH N. 11

NCTM. (2015). De los principios a la acción para garantizar el éxito matemático para todos. NCTM: México.

Rosenthal, R. (1994). Parametric measures of effect size. En H. Cooper \& L.V. Hedges (Eds.).The Handbook of Research Synthesis. pp. 231-260. Russell Sage Foundation: New York.
Shulman, L. S. (1986). Those who understand: knowledge growth in teaching. Educational Research. 4-14. SEP (2012). Acuerdo 592 para la articulación de la educación básica. México. 